\title{
Surface and bulk Landau levels in thin films of Weyl semimetals
}

\author{
Enrique Benito-Matías, ${ }^{*}$ Rafael A. Molina, ${ }^{\dagger}$ and José González \\ Instituto de Estructura de la Materia, CSIC, Serrano 123, E-28006 Madrid, Spain
}

(Received 24 July 2019; accepted 4 February 2020; published 24 February 2020)

\begin{abstract}
We show that the thin films of Weyl semimetals have a regime in which they develop an ordered sequence of Landau bands where the holelike levels are disentangled from their electronlike counterparts. We stress that this is not, however, a generic situation since in some cases there may be an inversion of Landau bands with electronand holelike character, preventing a clean observation of conductance quantization. Furthermore, we observe that two different types of Landau states may arise in thin films under perpendicular magnetic field, depending on whether the line connecting a pair of opposite Weyl nodes is parallel or perpendicular to the direction of the field. In the latter instance, we show that the flat Landau bands are made of states peaked at the two faces of the thin film. When the line connecting the Weyl nodes is parallel to the magnetic field, we see instead that the states in the Landau bands take the form of stationary waves with significant amplitude across the bulk of the material. In the regime with the ordered sequence of Landau bands, the states in the flat domains are confined along longitudinal sections of the thin film, and they start to disperse as long as the longitudinal propagation becomes close to the edges of the film, leading to edge states with distinctive profiles at the lateral boundaries for the two different types of Hall effect.
\end{abstract}

DOI: 10.1103/PhysRevB.101.085420

\section{INTRODUCTION}

In recent years, we have seen the discovery of different three-dimensional (3D) semimetals in which the Fermi surface is made of a number of nodes endowed with topological protection [1-8]. The Weyl semimetals (WSs) are unique in their class, as they have Weyl nodes acting like monopole charges of Berry curvature in momentum space. This is at the origin of the so-called chiral anomaly, which leads to the imbalance of the electronic charge in opposite Weyl nodes under suitable electric and magnetic fields.

Recently, the WSs have been investigated in the presence of a strong magnetic field, looking for signatures of the quantum Hall effect [9-14]. From a theoretical perspective, it has been shown that the 3D nodal-line semimetals have a regime in which they may host a rich structure of flat Landau bands, which are topologically protected as long as the particle-hole symmetry is preserved [15]. The discussion of the effect of a strong magnetic field on the 3D WSs is, however, more delicate since in some cases there may be an inversion of the Landau bands with electron- and holelike characters. Moreover, the relative orientation of the line connecting opposite Weyl nodes may play an important role in transport properties.

Thus, when the line joining opposite Weyl nodes and the magnetic field are parallel, the Landau bands keep a definite chirality like in the original model [11], but in the case where their directions are perpendicular, Landau levels of different chiralities are mixed for strong magnetic field, and a gap opens up. This destruction of the Weyl nodes has been observed

\footnotetext{
*enriquebenito2000@gmail.com

${ }^{\dagger}$ rafael.molina@csic.es

¥j.gonzalez@csic.es
}

in experiments for the WSs $\mathrm{TaP}$ and TaAs at very high magnetic fields fully in the quantum limit [16-18]. On the other hand, confirmation of a width-dependent quantum Hall transport has been achieved only by a carefully tuned wedge geometry in thin films of the Dirac semimetal $\mathrm{Cd}_{3} \mathrm{As}_{2}$ [19], when the angle between the line of nodes and the magnetic field was intermediate between the parallel and perpendicular configurations.

Anyhow, we stress that one of the main difficulties to observe the quantum Hall effect in thin films of WSs is that, in some cases, the Landau levels with holelike character may be intercalated among those with electronlike character in the low-energy part of the spectrum. When this happens, the linear branches of edge states dispersing upwards in energy may have multiple crossings with linear branches dispersing downwards, which may prevent a clean observation of conductance quantization of electronic transport from the edge states.

The best way to understand that phenomenon is to think of WSs which arise from a parent nodal-line semimetal where the nodal ring is gapped out by a suitable perturbation. Thus, when spin-orbit coupling is considered, band repulsion appears in the nodal line but only at nonzero values of the momenta $[8,20]$. Depending on the remaining symmetries and, in particular, the crystal symmetries, several Weyl or Dirac points may remain after including spin-orbit effects. The value of the spin-orbit coupling, which depends on the mass of the atoms forming the material, controls the transition between these two different regimes. This is a very generic scenario that has been proposed for the TaAs [21], ZrTe [8], and $\mathrm{Cu}_{3}(\mathrm{Pd}, \mathrm{Zn}) \mathrm{N}[22,23]$ families of materials, among others.

We will show that, when the energy scale of the inverted bands of the parent nodal-line semimetal is much larger than 


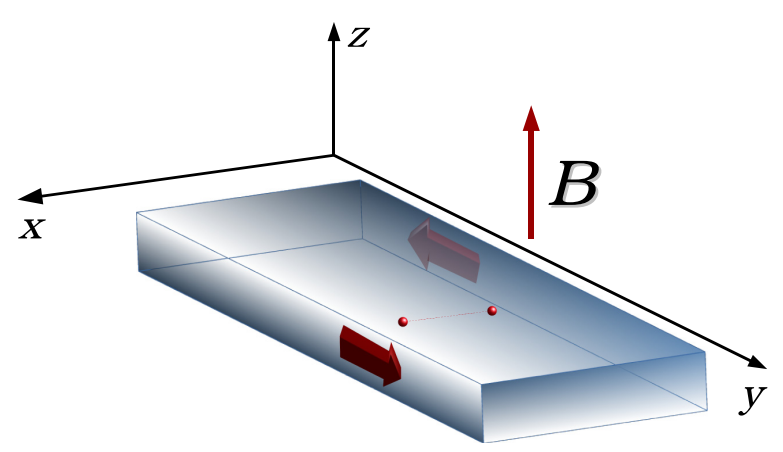

(a)

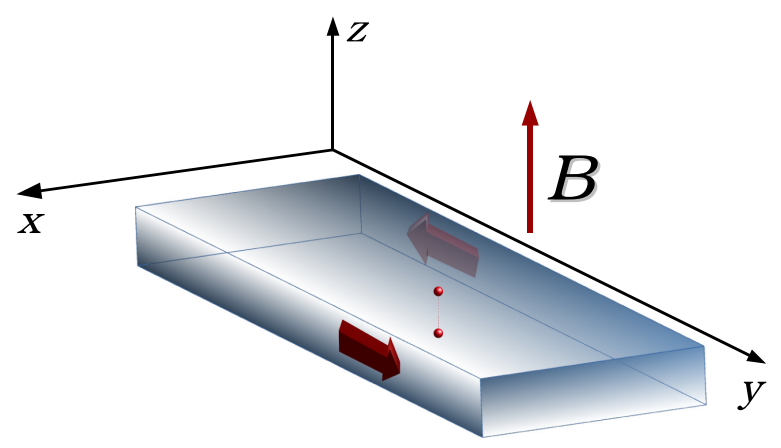

(b)

FIG. 1. Schematic view of the geometry of the Hall bar considered in the paper, with finite width in the $x$ direction and infinite dimension in the longitudinal $y$ direction. The different cases in (a) and (b) correspond to setups with the line connecting the Weyl points (large dots) being perpendicular and parallel to the transverse magnetic field, respectively.

the scale of the perturbation, the system is in a regime with a disordered intercalation of Landau bands with electron- and holelike character. In what follows, we consider a simple model of WS which will allow us to illustrate precisely this situation, which we denominate the inversion regime. On the other hand, the opposite case is faced in WSs with sufficiently steep linear dispersion, which leads then to a complete separation in energy between electron- and holelike Landau bands. We will also illustrate more precisely this instance in what follows, referring to it as the Weyl-cone regime (in opposition to the inversion regime).

We identify then a regime of parameters of the WSs (actually relevant for the study of real materials) in which the thin-film geometries develop an ordered sequence of Landau bands where the holelike levels are disentangled from their electronlike counterparts. Addressing the case of thin films with the setup represented in Fig. 1, we will see that two different types of Landau states may arise depending on whether the line connecting a pair of opposite Weyl nodes is parallel or perpendicular to the direction of the magnetic field. In the latter instance, where there are Fermi arcs arising from the projection of the line connecting the Weyl nodes onto the surface of the semimetal, we will show that the flat Landau levels are made of states peaked at the two faces of the thin film [24]. These may be considered the counterpart, in a full quantum-mechanical picture, of the semiclassical cyclotron orbits connecting opposite Fermi arcs [10,13,25]. When the line connecting the Weyl nodes is parallel to the direction of the magnetic field, we will see instead that the states in the Landau levels take the form of stationary waves with significant amplitude across the bulk of the material.

In the Weyl-cone regime characterized below, we will show that the states of the thin film follow a pattern of quantization in a strong magnetic field which is very similar to that of the two-dimensional (2D) quantum Hall effect. In the geometry of Fig. 1, that quantization corresponds to the confinement of the states along longitudinal sections, propagating in opposite directions as they approach opposite edges of the bar. Such a localization is indeed behind the existence of flat Landau bands, which start to disperse as long as the longitudinal propagation becomes close to the edges of the film, leading to edge states with distinctive profiles at the lateral boundaries for the two different types of Hall effect in the WS.

\section{LANDAU LEVELS FROM SURFACE STATES}

We first consider the case in which the line connecting opposite Weyl nodes is parallel to the surface of the thin film. In this setup, that line has a projection onto the two faces of the material, with the consequent formation of surface Fermi arcs. Then, it becomes interesting to find out about the role of the surface states in the quantum Hall regime. In this regard, we are going to see that the low-energy Landau levels in the thin film are not made of Landau states of the 3D material, so that they can be found only by investigating the finite geometry.

We first take the Weyl nodes aligned along the $x$ direction in the geometry of Fig. 1. The magnetic field points in the $z$ direction, and we choose the gauge in which the vector potential is $\mathbf{A}=(0, B x, 0)$. We then model the WS with the Hamiltonian

$$
\begin{aligned}
H_{1}= & \left\{m_{0}+m_{1}\left[\partial_{x}^{2}-\left(-i \partial_{y}+B x\right)^{2}+\partial_{z}^{2}\right]\right\} \sigma_{z} \\
& -i v \sigma_{x} \partial_{z}+v \sigma_{y}\left(-i \partial_{y}+B x\right) .
\end{aligned}
$$

In this case, we can partially diagonalize the Hamiltonian by introducing creation and annihilation operators of the modes of a harmonic oscillator

$$
\begin{aligned}
& a=\frac{1}{\sqrt{2}}\left(\sqrt{B}\left(x+k_{y} / B\right)+\frac{\partial_{x}}{\sqrt{B}}\right), \\
& a^{\dagger}=\frac{1}{\sqrt{2}}\left(\sqrt{B}\left(x+k_{y} / B\right)-\frac{\partial_{x}}{\sqrt{B}}\right) .
\end{aligned}
$$

This brings the Hamiltonian to the form [26]

$$
\begin{aligned}
H_{1}= & \left\{m_{0}+m_{1}\left[-2 B\left(a^{\dagger} a+1 / 2\right)+\partial_{z}^{2}\right]\right\} \sigma_{z} \\
& -i v \sigma_{x} \partial_{z}+v \sqrt{\frac{B}{2}} \sigma_{y}\left(a+a^{\dagger}\right) .
\end{aligned}
$$

\section{A. Thin-film versus 3D regime of Weyl semimetals}

If we apply the Hamiltonian (4) to the case of the thin film with faces parallel to the $x-y$ plane, we can already see that the Landau states cannot take the form of simple stationary waves along the $z$ direction. This becomes clear from inspection of the dependence on $\partial_{z}$, which implies that the form of the spinor wave function vanishing at one of the faces of the film 

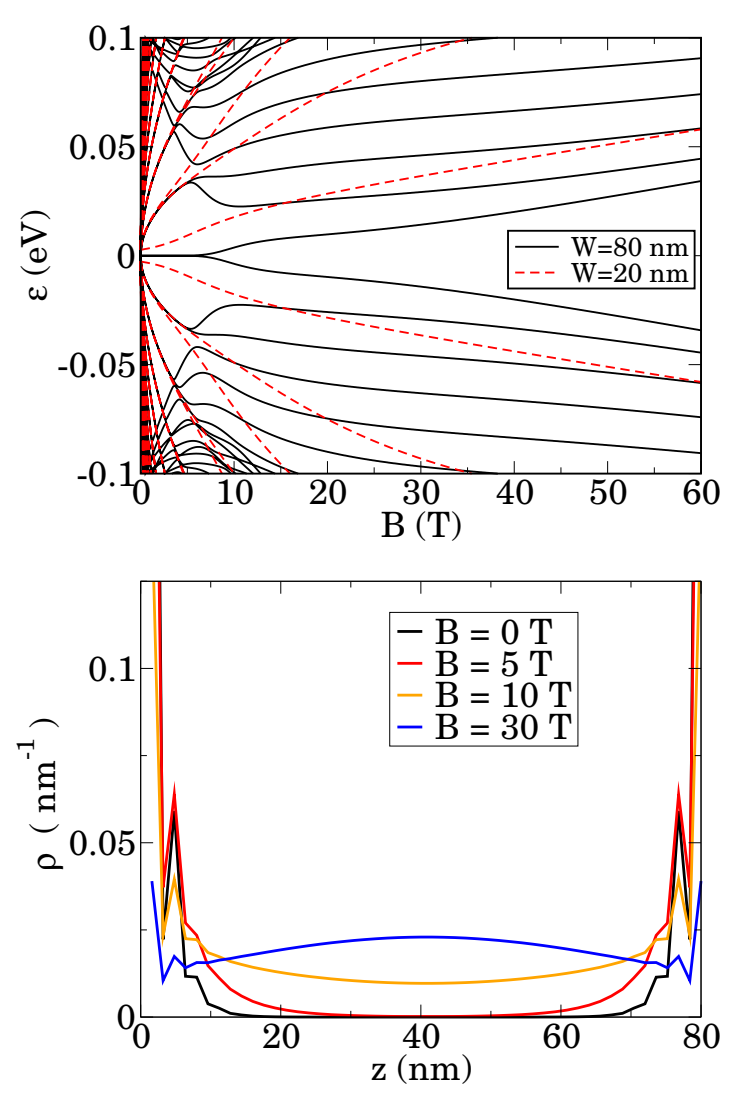

FIG. 2. Top: Energy bands of a slab of WS in a perpendicular magnetic field as a function of the magnetic field. The parameters are $m_{0}=0.1 \mathrm{eV}, m_{1}=0.2 \mathrm{eV} \mathrm{nm}^{2}$, and $v=0.5 \mathrm{eV} \mathrm{nm}$. The red curves correspond to a depth of $W=20 \mathrm{~nm}$ in the $z$ direction, while the black curves correspond to $W=80 \mathrm{~nm}$. Bottom: Electronic density in the $z$ direction of the state with positive energy closer to the band center for the case $W=80 \mathrm{~nm}$ and magnetic field $B=0,5,10,30 \mathrm{~T}$.

(say, at $z=W / 2$ ) cannot match the form of the spinor wave function vanishing at the opposite face (say, at $z=-W / 2$ ). Yet it can be shown that the eigenstates of the Hamiltonian (4) are arranged in flat Landau levels, which develop from the surface states in the Fermi arcs of the WS.

In a slab geometry with some finite depth in the $z$ direction and infinite in the $x$ and $y$ directions, the Hamiltonian in Eq. (4) can be diagonalized using a real-space discretization in the $z$ direction and a cutoff in the number of coupled Landau modes considered. In this geometry, as $k_{y}$ is a good quantum number and $x$ is infinite, the Landau levels present a high degeneracy that shows clearly in the definition of the creation and destruction operators, Eqs. (2) and (3). All possible values of $k_{y}$ have the same energy within each Landau band.

We show in the top panel of Fig. 2 the dependence of the energy of the states with respect to the magnetic field (the Landau fan) for two different values of the depth of the slab $W=20 \mathrm{~nm}$ and $W=80 \mathrm{~nm}$. In the narrower slab, the different states in Fig. 2 come from the Landau levels of the surface states. As there is a large hybridization between the two surfaces, a very noticeable gap forms between the electron and hole sides of the spectrum for all values of $B$.

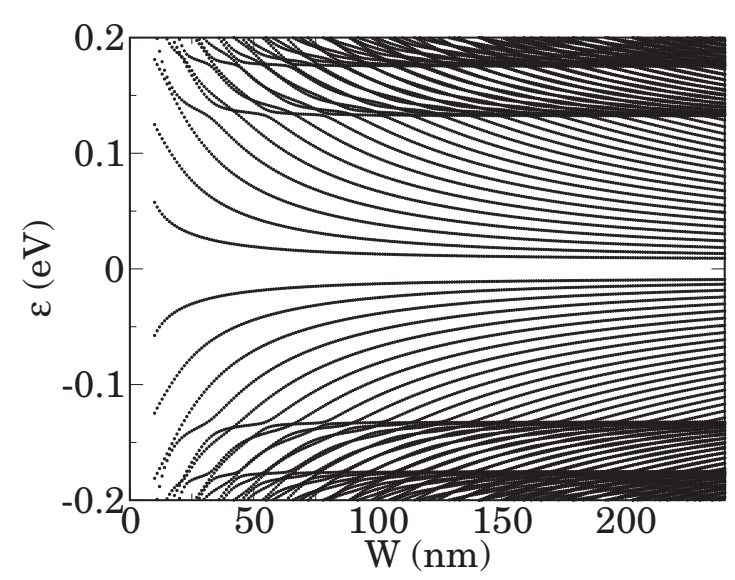

FIG. 3. Energy bands of a slab of WS in a perpendicular magnetic field as a function of the depth in the $z$ direction for $B=30 \mathrm{~T}$. The parameters are the same as in Fig. 2.

In the thicker slab, the hybridization is negligible, and the gap is close to zero up to a finite value of the magnetic field. The Landau states coming from the bulk mix with the Landau states coming from the surface, producing a number of avoided crossings. The mixing with bulk states opens a gap for magnetic fields larger than $10 \mathrm{~T}$. The effect of mixing with the bulk is seen in Fig. 2 for the electronic density of the state closer to zero energy as the magnetic field is increased (bottom panel).

From a practical point of view, there is a clear distinction between the thin-film regime and the 3D limit of WSs in that the former displays a clear separation in energy between surface and bulk states in the low-energy part of the spectrum [27]. We represent the transition between both regimes in Fig. 3, where we plot the dependence of the bands on the depth $W$ of the slab in the $z$ direction for a magnetic field of $B=$ $30 \mathrm{~T}$. The gap in the middle of the band structure saturates for $W>200 \mathrm{~nm}$, while the typical separation between the levels above the gap scales as $1 / W$.

\section{B. Inversion versus Weyl-cone regime of Weyl semimetals}

In the case of a bar with finite width in the $x$ direction, the energy bands are flat in a certain range of the momentum $k_{y}$, but for larger values they become dispersive as the Landau states approach the boundaries of the bar. In order to compute in this geometry, we use a real-space discretization of Hamiltonian (1) in both the $x$ and $z$ directions with hard-wall boundary conditions [leading to wave functions such that $\psi( \pm L / 2, y, z)=\psi(x, y, \pm W / 2)=0]$.

It turns out that not all the choices of parameters in the Hamiltonian (1) lead to a system where the transport from the edge states can be measured in a clean way. In an ideal situation, the levels of electron states must be well separated from the levels of holes, so that only electron states (or holes states) contribute to transport for a given position of the Fermi level. This cannot be guaranteed when $m_{0}-m_{1} B \gg v \sqrt{B}$ since in that case the electronlike levels may cross at multiple points with the holelike levels as a consequence of the change in sign of the quantity inside curly brackets in (1) as the order of the Landau level increases. The outcome of such multiple 


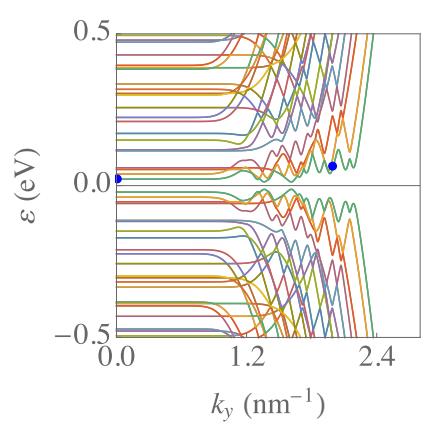

(a)

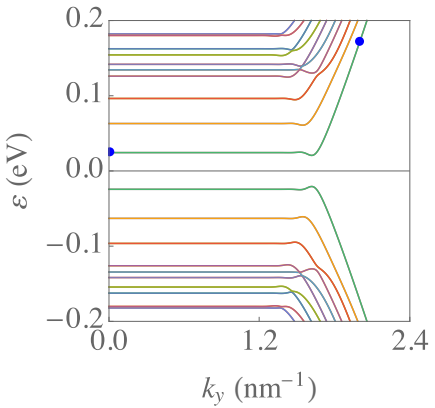

(b)
FIG. 4. Energy bands of a thin film of WS in a perpendicular magnetic field in a setup with the line connecting opposite Weyl nodes parallel to the faces of the film. The levels have been computed for a bar with transverse dimension $L=80 \mathrm{~nm}$ and depth $W=20 \mathrm{~nm}$ under a magnetic field of $B=30 \mathrm{~T}$. The parameters of the model of WS corresponding to each plot are (a) $m_{0}=1.0 \mathrm{eV}$, $m_{1}=2.0 \mathrm{eV} \mathrm{nm}{ }^{2}, v=0.1 \mathrm{eV} \mathrm{nm}$ [with $\left(m_{0}-m_{1} B\right) / v \sqrt{B} \simeq 42.62$ ] and (b) $m_{0}=0.1 \mathrm{eV}, m_{1}=0.2 \mathrm{eV} \mathrm{nm}{ }^{2}, v=0.5 \mathrm{eV} \mathrm{nm}$ [with $\left(m_{0}-\right.$ $\left.\left.m_{1} B\right) / v \sqrt{B} \simeq 0.85\right]$. Blue dots correspond to the states whose profiles are shown in Fig. 7.

crossings can be observed in Fig. 4(a). This illustrates what we will call the inversion regime since it corresponds to the case where the behavior of the levels is dominated by the inversion of the bands about the nodal line when $v \rightarrow 0$.

On the other hand, we find the opposite situation when $m_{0}-m_{1} B \ll v \sqrt{B}$, which we may call the Weyl-cone regime as the Landau levels develop then essentially from the linear dispersion of the Weyl cones. Taking into account that the Landau states are mainly attached in this case to the surface of the thin film, the Landau levels can be understood as being typical of effective Dirac fermions with a gap (mass) deriving from the $\sigma_{z}$ term in the Hamiltonian. This can be seen in Fig. 4(b), which represents a characteristic situation with almost complete separation between electron- and holelike levels.

The significance of being in one regime or the other becomes apparent when looking at the dispersive branches in the geometry shown in Fig. 1. In the regime with $m_{0}-m_{1} B \gg$ $v \sqrt{B}$, the inversion between electron- and holelike Landau bands gives rise to multiple crossings between linear branches of electronlike edge states dispersing upwards and holelike edge states dispersing downwards. In a situation like that shown in Fig. 4(a), any choice of Fermi level may cut many linear branches, each contributing to the current $j_{y}=\partial \varepsilon / \partial k_{y}$. The problem is that these contributions come from states propagating in opposite directions and, in general, away from the edge of the bar. We recall that the electric current $I_{y}$ can be expressed as an integral over the filled states (reinstating, at this point, $\hbar$ in the equations),

$$
I_{y}=\frac{e}{\hbar} \int_{\text {filled states }} \frac{d k_{y}}{2 \pi} \frac{\partial \varepsilon}{\partial k_{y}} .
$$

This electric current becomes proportional to the difference between the respective chemical potentials $\varepsilon_{+}, \varepsilon_{-}$at opposite edges of the bar only if the boundaries of the integral can be mapped to the spatial lateral boundaries. This happens in the

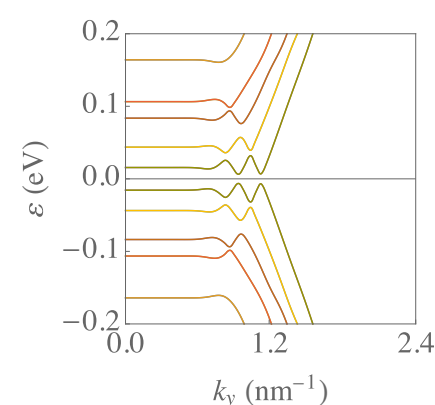

(a)

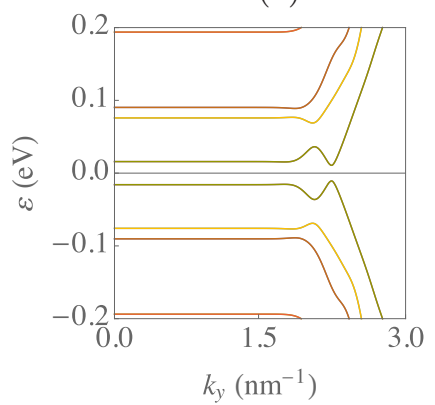

(c)

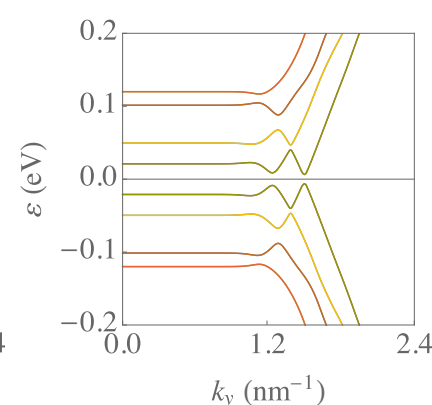

(b)

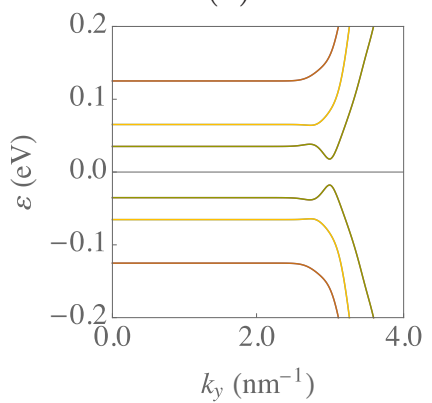

(d)
FIG. 5. Energy bands of a thin film of WS in a perpendicular magnetic field in a setup with the line connecting opposite Weyl nodes parallel to the faces of the film. The levels have been computed for a bar with transverse dimension $L=60 \mathrm{~nm}$ and depth $W=$ $20 \mathrm{~nm}$, and values of the magnetic field, from (a) to (d), equal to $30,40,60$, and $80 \mathrm{~T}$. The parameters used to model the WS are $m_{0}=0.8 \mathrm{eV}, m_{1}=1.6 \mathrm{eV} \mathrm{nm}{ }^{2}, v=0.5 \mathrm{eV} \mathrm{nm}$. The quantity $\left(m_{0}-m_{1} B\right) / v \sqrt{B}$ equals, from (a) to (d), $\approx 6.82,5.71,4.34,3.48$.

ordered sequence of Landau bands represented in Fig. 4(b), where the integral is proportional to the number $n$ of linear branches picked up at the boundaries, leading to the usual quantization rule of the Hall conductance $G=n\left(e^{2} / h\right)$.

One can check, however, that the multiple crossings may be cured by suitably increasing the magnetic field. This can be seen in Fig. 5, which shows the Landau levels for a WS with parameters placing the system originally in the inversion regime. As the magnetic field is increased from 30 to $80 \mathrm{~T}$, we observe that the electronlike levels are disentangled from the holelike levels. This is consistent with the fact that it is the magnitude of $m_{0}-m_{1} B$ (compared to $v \sqrt{B}$ ) which actually dictates whether the WS is in the inversion regime or not.

Anyhow, the ideal regime to observe the current quantization along the edge (and conductance quantization in the quantum Hall effect) is that of WSs with $m_{0}-m_{1} B \ll v \sqrt{B}$. This is illustrated in Fig. 6, which represents the Landau levels for a sequence of WSs passing from the inversion to the Weyl-cone regime as the parameter $m_{0}$ is decreased. In the final stage, when $v \sqrt{B}$ becomes larger than $m_{0}-m_{1} B$, we observe that the electronlike bands (with linear branches dispersing upwards) become well separated from the holelike bands (with linear branches dispersing downwards).

\section{Profile of Landau states}

When the WS is in the regime with $v \sqrt{B}$ larger than $m_{0}-$ $m_{1} B$, the Landau states follow a clear pattern of localization 


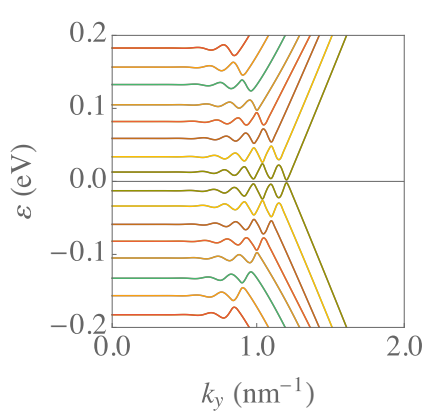

(a)

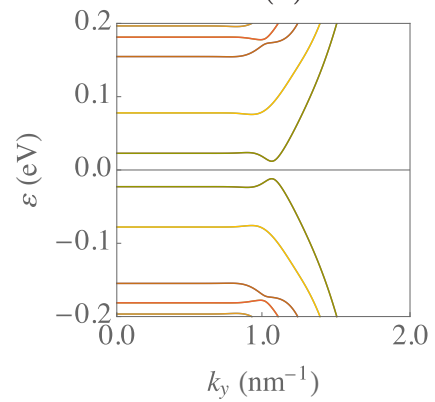

(c)

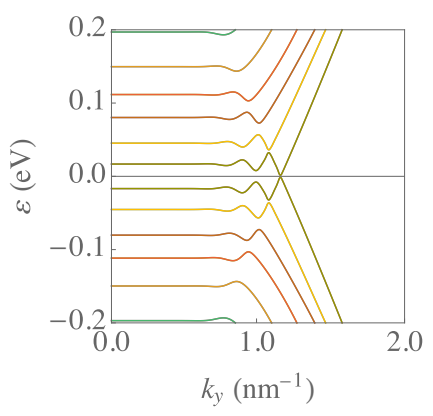

(b)

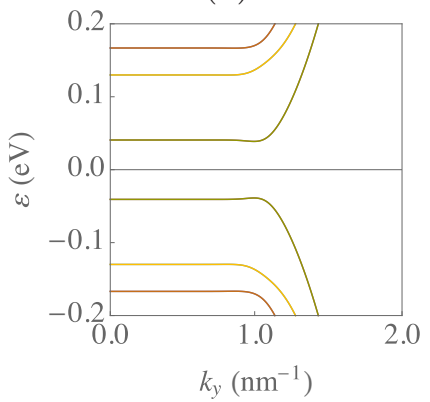

(d)

FIG. 6. Energy bands of a thin film of WS in a perpendicular magnetic field in a setup with the line connecting opposite Weyl nodes parallel to the faces of the film. The levels have been computed for a bar with transverse dimension $L=60 \mathrm{~nm}$, depth $W=20 \mathrm{~nm}$, and magnetic field $B=30 \mathrm{~T}$. The model of WS has parameters $m_{1}=1.0 \mathrm{eV} \mathrm{nm}{ }^{2}, v=0.5 \mathrm{eV} \mathrm{nm}$, and $m_{0}$ taking values, from (a) to (d), equal to $1.2,0.6,0.2$, and $0.1 \mathrm{eV}$ [leading, respectively, to $\left.\left(m_{0}-m_{1} B\right) / v \sqrt{B} \simeq 10.82,5.2,1.45,0.51\right]$.

in the geometry represented in Fig. 1. We may consider for concreteness the model of a WS with the parameters leading to the Landau levels shown in Fig. 4(b). When $k_{y}$ is in the flat domain of the Landau level, the corresponding states are localized in longitudinal sections (along the $y$ direction) away from the lateral boundaries of the bar [see Fig. 7(a)]. As $k_{y}$ approaches the dispersive regime of the bands, the longitudinal states become progressively closer to the edges of the bar, as shown in Fig. 7(b). These profiles can be compared with their counterparts in the inversion regime. There, it can be observed that the wave functions behave qualitatively in the same way as a function of $k_{y}$ [Figs. 7(c) and 7(d)] although the dispersive bands tend to delocalize the states away from the edge, as shown in Fig. 7(d).

On the other hand, the shape of the probability density along the depth of the film is also remarkable. For the states which propagate longitudinally far from the edges of the bar, the probability density is peaked close to each face of the thin film, but with a profile which does not decay completely in the interior of the film [see Fig. 8(a)]. This distribution changes drastically when the states approach the edges of the bar since then the probability density accumulates around one of the faces of the film depending on the sign of $k_{y}$, as shown in Fig. 8(b).

The accumulation of the edge states in only one of the faces of the film is indeed characteristic of setups in which the Weyl nodes are not aligned with the longitudinal direction of the

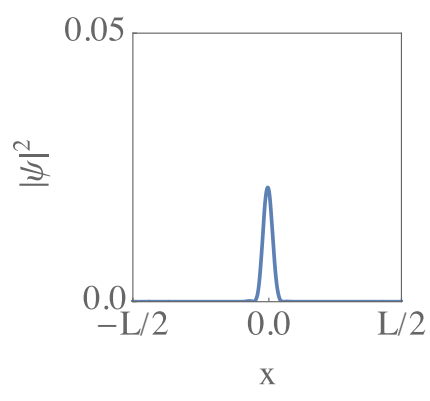

(a)

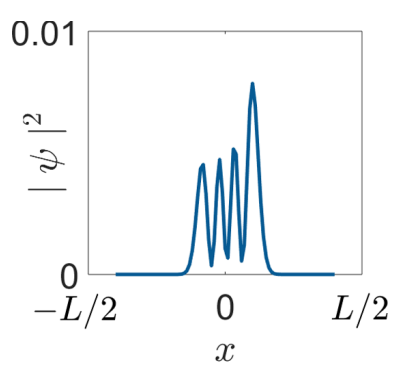

(c)

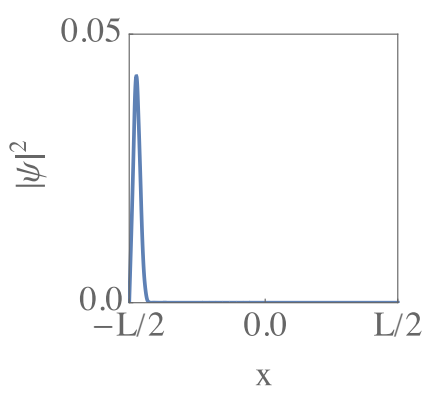

(b)

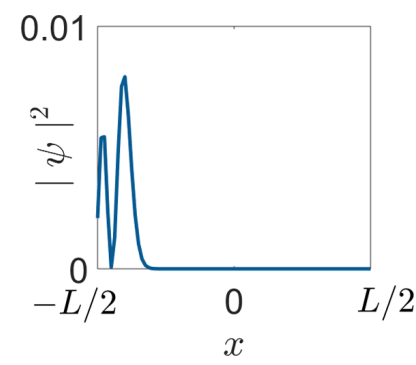

(d)
FIG. 7. (a) and (b) Profiles of the probability density along the transverse dimension of a thin film for two different eigenstates in the band with the lowest positive energy represented in Fig. 4(b) (Weylcone regime), corresponding to (a) $k_{y}=0$ and (b) $k_{y}=2.0 \mathrm{~nm}^{-1}$. The profiles are taken at a depth of $3 \mathrm{~nm}$ from the upper surface of the thin film. (c) and (d) Profiles for the same momenta as in (a) and (b) of the respective states in the inversion regime of Fig. 4(a).

bar, which is the case considered in the above discussion. If we change, however, the orientation of the bar so that its longitudinal dimension becomes parallel to the line connecting the Weyl nodes, then the edge states acquire an even probability density along the depth of the film. In this particular case, the electron density decays drastically inside the film, but it is clearly accumulated in the interior when the electrons run close to the edge of the bar, as can be seen in Fig. 9.

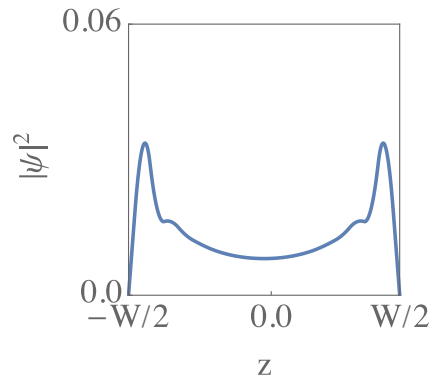

(a)

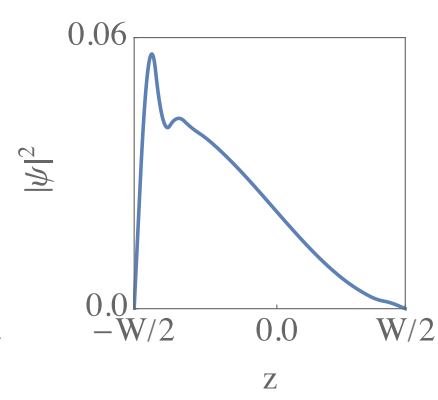

(b)
FIG. 8. Profiles of probability density for the same states considered in Figs. 7(a) and 7(b) but taken now along the depth of the thin film for respective sections (a) at the center of the bar $x=0$ and (b) at a distance $x$ of $3 \mathrm{~nm}$ from the edge. The longitudinal dimension of the bar is perpendicular to the line connecting the Weyl nodes as in Fig. 7. 


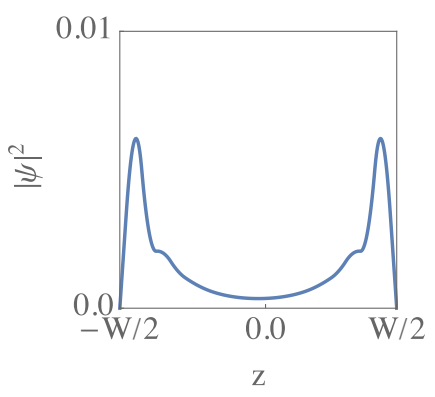

(a)

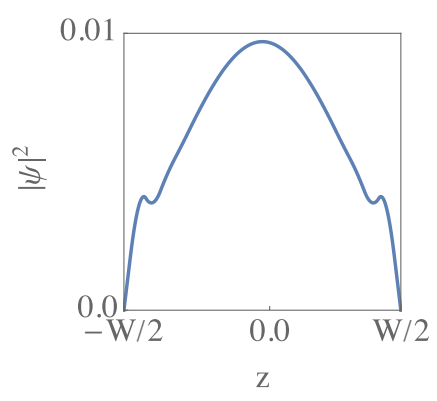

(b)
FIG. 9. Profiles of the probability density along the depth of a bar for two eigenstates of WS with the same geometry and parameters as in Fig. 8, but with the longitudinal dimension of the bar running parallel to the line connecting the Weyl nodes. The profiles are taken for respective sections (a) at the center of the bar $x=0$ and (b) at a distance $x$ of $3 \mathrm{~nm}$ from the edge.

\section{LANDAU LEVELS FROM BULK STATES}

We discuss now the setup in which the line connecting the Weyl nodes is perpendicular to the faces of the thin film. In this case, the projection of that line onto the surface of the material reduces to a point, so that no contribution to transport can be expected from surface states.

We take the Weyl nodes aligned along the $z$ direction in the setup of Fig. 1, and a magnetic field perpendicular to the thin film with vector potential $\mathbf{A}=(0, B x, 0)$. Then we model the WS with the Hamiltonian

$$
\begin{aligned}
H_{2}= & \left\{m_{0}+m_{1}\left[\partial_{x}^{2}-\left(-i \partial_{y}+B x\right)^{2}+\partial_{z}^{2}\right]\right\} \sigma_{z} \\
& -i v \sigma_{x} \partial_{x}+v \sigma_{y}\left(-i \partial_{y}+B x\right) .
\end{aligned}
$$

Making use of operators (2) and (3), we can partially diagonalize the Hamiltonian, which takes the form [11]

$$
\begin{aligned}
H_{2}= & \left\{m_{0}+m_{1}\left[-2 B\left(a^{\dagger} a+1 / 2\right)+\partial_{z}^{2}\right]\right\} \sigma_{z} \\
& -i v \sqrt{\frac{B}{2}} \sigma_{x}\left(a-a^{\dagger}\right)+v \sqrt{\frac{B}{2}} \sigma_{y}\left(a+a^{\dagger}\right) .
\end{aligned}
$$

The main difference with respect to the discussion in the previous section is that now one can find eigenstates of (7) vanishing at both surfaces of the thin film while keeping the same form of the spinor from one face to the other. This means that the wave functions take the simple form of stationary waves inside the film, with well-defined values of the momentum $k_{z}$ in the $z$ direction. Thus, contrary to the case of the setup considered in the previous section, the eigenstates are not attached in the present situation to the faces of the thin film, but they are extended over its interior. This simplifies the resolution of the eigenvalue problem, which can be carried out analytically, as reported in Ref. [11]

Anyhow, the point we want to make is that there is again a regime of parameters in which the eigenstates of (7) can be arranged into an ordered sequence of Landau bands in a sufficiently strong magnetic field. The Weyl-cone and inversion regimes are exactly the same as in the previous section since the expression between curly brackets in the Hamiltonian (7) may change sign in the same way as it does in (4) when the order of the Landau level increases.

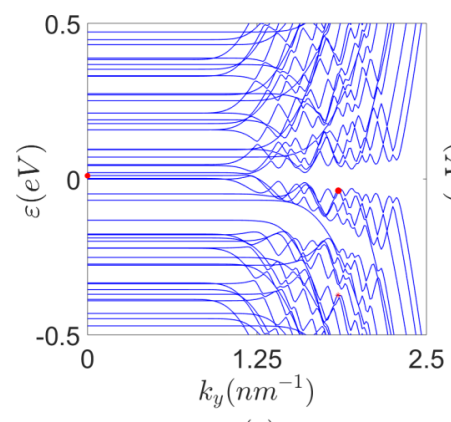

(a)

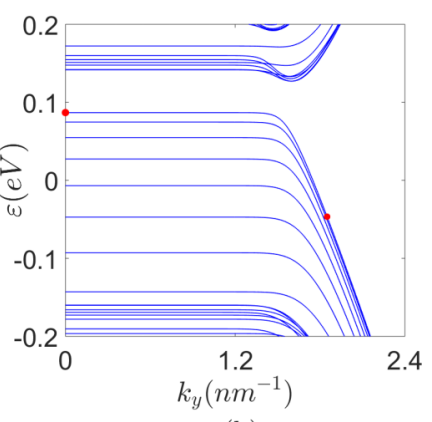

(b)
FIG. 10. Energy bands of a thin film of WS in a setup with magnetic field parallel to the line connecting opposite Weyl nodes and perpendicular to the faces of the film. Calculations are made in a bar with transverse dimension $L=80 \mathrm{~nm}$ and depth $W=$ $20 \mathrm{~nm}$ under a magnetic field of $B=30 \mathrm{~T}$. Model parameters are (a) $m_{0}=1.0 \mathrm{eV}, m_{1}=2.0 \mathrm{eV} \mathrm{nm}{ }^{2}, v=0.1 \mathrm{eVnm}\left[\left(m_{0}-\right.\right.$ $\left.\left.m_{1} B\right) / v \sqrt{B} \simeq 42.62\right]$ and (b) $m_{0}=0.1 \mathrm{eV}, m_{1}=0.2 \mathrm{eV} \mathrm{nm}{ }^{2}, v=$ $0.5 \mathrm{eVnm}\left[\left(m_{0}-m_{1} B\right) / v \sqrt{B} \simeq 0.85\right]$. Red marks indicate the energy-momentum coordinates for the states shown in Figs. 13 and 14 .

We compute levels and wave functions in a bar geometry with a real-space discretization of Hamiltonian (6) along the $x$ and $z$ directions with hard-wall boundary conditions. Figure 10 shows the bands for the model parameters previously considered in Fig. 4. Figure 11 shows the transition from the inversion regime to the Weyl-cone regime as a function of the magnetic field for a given choice of model parameters, and Fig. 12 illustrates this transition for fixed magnetic field and decreasing values of the $m_{0}$ parameter. As can be seen
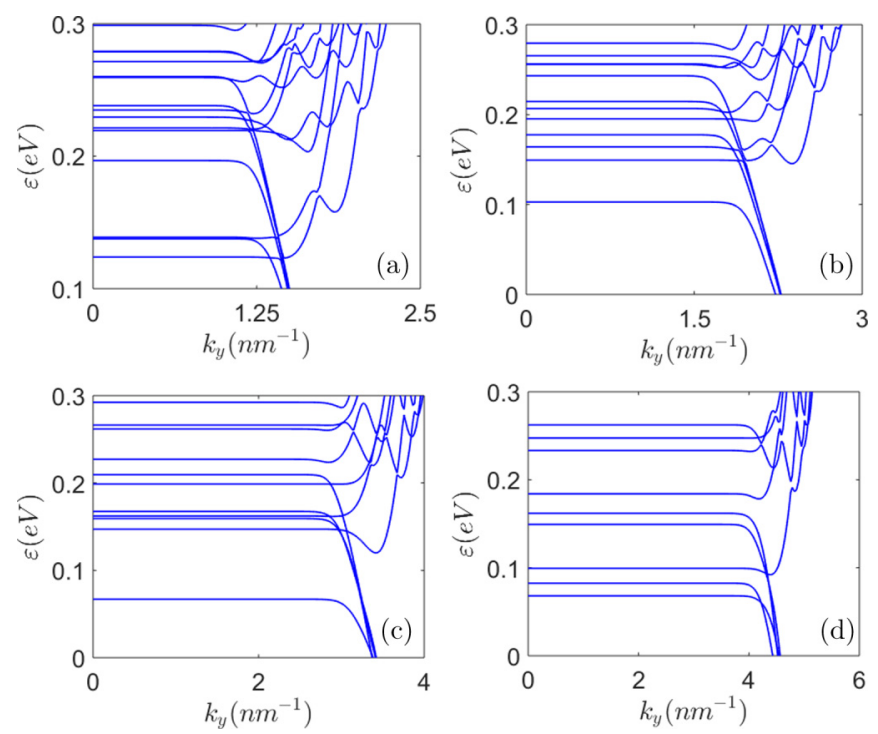

FIG. 11. Energy bands of a thin film of WS in a setup with magnetic field parallel to the line connecting opposite Weyl nodes and perpendicular to the faces of the film. The levels have been computed for a bar with transverse dimension $L=80 \mathrm{~nm}$ and depth $W=20 \mathrm{~nm}$ and values of the magnetic field, from (a) to (d), equal to $30,40,60$, and $80 \mathrm{~T}$. The parameters used to model the WS are $m_{0}=0.8 \mathrm{eV}, m_{1}=1.6 \mathrm{eV} \mathrm{nm}{ }^{2}, v=0.5 \mathrm{eV} \mathrm{nm}$. The quantity $\left(m_{0}-m_{1} B\right) / v \sqrt{B}$ equals, from (a) to $(\mathrm{d}), \approx 6.82,5.71,4.34,3.48$. 

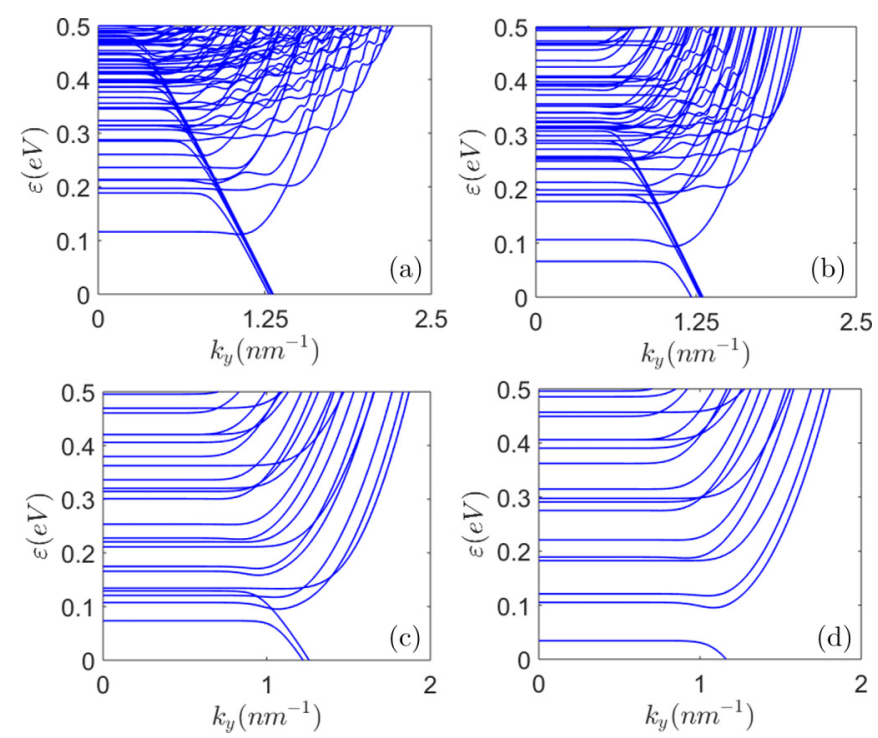

FIG. 12. Energy bands of a thin film of WS in a setup with magnetic field parallel to the line connecting opposite Weyl nodes and perpendicular to the faces of the film. The levels have been computed for a bar with transverse dimension $L=60 \mathrm{~nm}$, depth $W=20 \mathrm{~nm}$, and magnetic field $B=30 \mathrm{~T}$. The model of WS has parameters $m_{1}=1.0 \mathrm{eV} \mathrm{nm}{ }^{2}, v=0.5 \mathrm{eV} \mathrm{nm}$, and $m_{0}$ taking values, from (a) to (d), equal to $1.2,0.6,0.2$, and $0.1 \mathrm{eV}\left[\left(m_{0}-m_{1} B\right) / v \sqrt{B} \simeq 10.82\right.$, $5.2,1.45,0.51$, respectively].

in Fig. 11, the transition from inversion to Weyl-cone regime with increasing magnetic field is not as clean, in general, as it was in the case with the line connecting opposite Weyl nodes perpendicular to the field. In this case, the effectiveness of the field to disentangle the electron from the holelike levels is strongly dependent on the model parameters.

The flat domain of the bands corresponds to eigenstates that are confined to longitudinal sections away from the edges of the bar. These bands start to get some dispersion as soon as the increase in $k_{y}$ drives the longitudinal propagation of the states close to the edges of the bar. This change in the localization along the transverse dimension can be seen in Fig. 13.

In this setup, the probability density of the states in a given Landau band keeps the same shape in the interior of the film, without much variation as the longitudinal propagation approaches the edge, as shown in Fig. 14, which is in accordance with the bulk character of the eigenstates.

\section{PERTURBATIONS BREAKING PARTICLE-HOLE SYMMETRY}

The above discussion is based on the Hamiltonians (1) and (6), which can be taken as a first approximation to model the WSs. This approach captures the low-energy physics of a pair of Weyl nodes, but the description of real materials has to be complemented with the effect of diagonal perturbations (that is, perturbations which are proportional to the identity in pseudospin space).

Real materials displaying WS behavior can be accurately modeled by adding to the Hamiltonians (1) and (6) new terms which are proportional to the square of the momentum. In
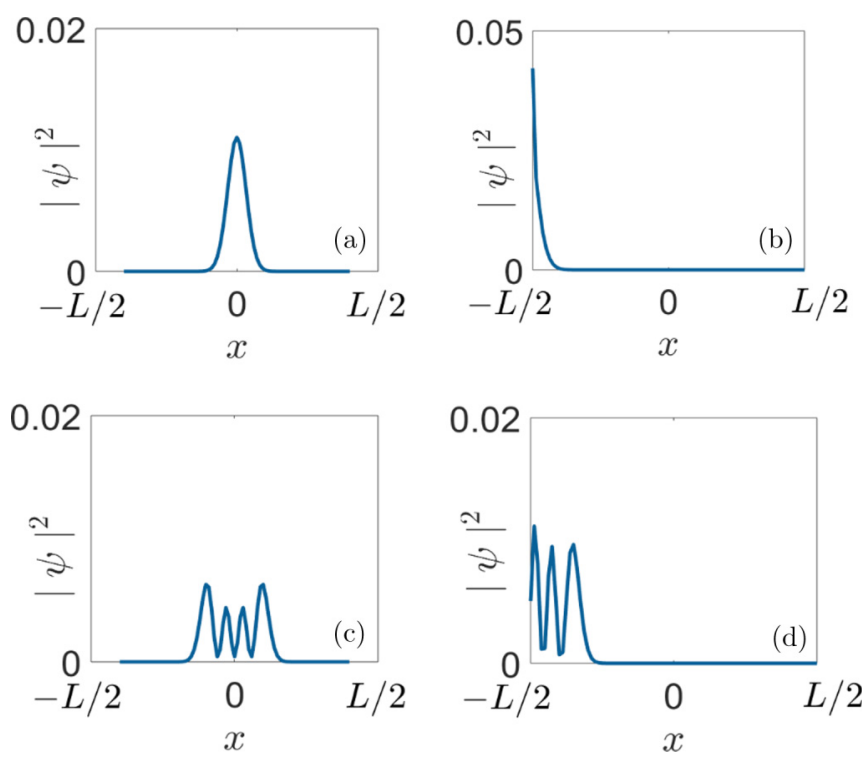

FIG. 13. (a) and (b) Profiles of the probability density for two different eigenstates in the highest holelike band below the gap in the band structure shown in Fig. 10(b) (Weyl-cone regime), corresponding to (a) $k_{y}=0$ and (b) $k_{y}=1.85 \mathrm{~nm}^{-1}$. The profiles are taken along the transverse dimension of the bar, at a depth of $10 \mathrm{~nm}$ from the surface. (c) and (d) Profiles for the same momenta as in (a) and (b) of the respective states in the inversion regime in Fig. 10(a).

order to assess the effect of these perturbations, we pursue the same analysis carried out before, but adding now to (1) and (6) a new term,

$$
\Delta H=a_{0}+a_{1}\left[\partial_{x}^{2}-\left(-i \partial_{y}+B x\right)^{2}+\partial_{z}^{2}\right] .
$$

The effect of $a_{0}$ is just to produce a rigid shift of all the energy levels, and it can be disregarded. However, the term introduced by $a_{1}$ has more significance, in particular because it breaks the particle-hole symmetry of the Hamiltonian (1)
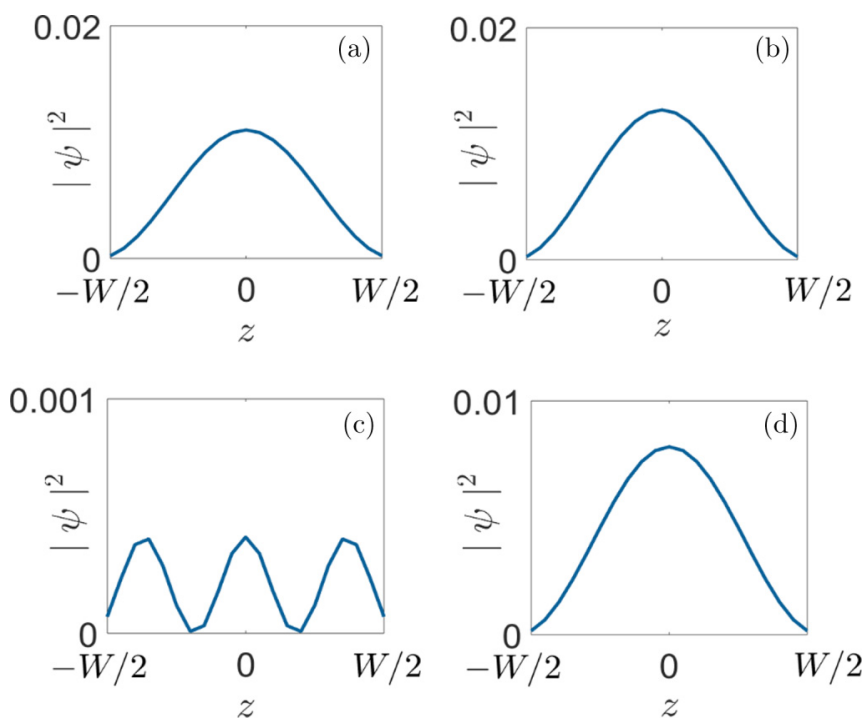

FIG. 14. Profiles of probability density for the same respective states considered in Fig. 13, but taken now along the depth of the thin film, for sections at the center of the bar $x=0$ (left plots) and at a distance $x$ of $2 \mathrm{~nm}$ from the edge (right plots). 


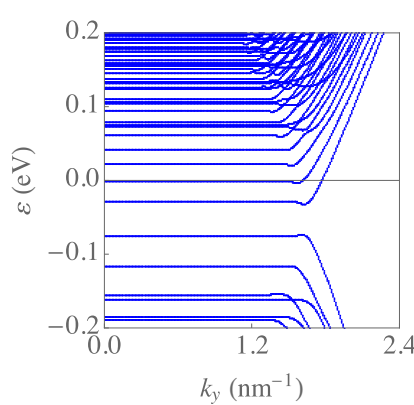

(a)

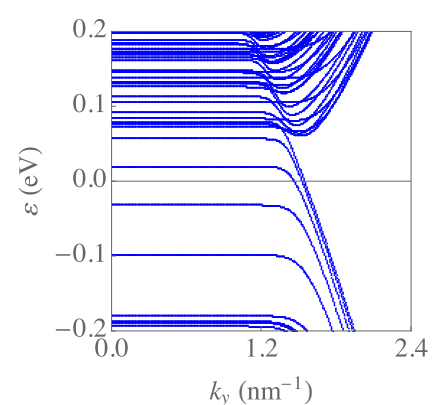

(b)
FIG. 15. Energy bands of a thin film of WS in a perpendicular magnetic field for the same respective setups and parameters considered in Figs. 4(b) and 10(b), with the line connecting opposite Weyl nodes perpendicular (parallel) to the magnetic field for the left (right) plot, but with the addition of diagonal terms in the Hamiltonian with $a_{0}=0$ and $a_{1}=0.1 \mathrm{eV} \mathrm{nm}^{2}$.

while enhancing the deviation from such a symmetry in the Hamiltonian (6).

In the case of a thin film with the line connecting the Weyl nodes along the $x$ direction in the setup of Fig. 1, the diagonalization of the Hamiltonian $H_{1}+\Delta H$ produces the band structure represented in Fig. 15(a). It can be seen that, for sufficiently small values of $a_{1}$, the original low-energy Landau levels shown in Fig. 4(b) can be clearly recognized in the band structure of the perturbed system. However, when $a_{1}$ goes beyond a certain threshold, the Landau levels start to accumulate in a very dense structure in the particle sector (for $a_{1}>0$ ), already incipient in Fig. 15(a).

The same effect of condensation of Landau levels can be seen in a thin film with the line connecting the Weyl nodes perpendicular to the surface of the film. In this case, the band structure obtained from the diagonalization of $H_{2}+\Delta H$ is represented in Fig. 15(b). The origin of the accumulation of the levels can be more clearly understood in this model since the momentum $k_{z}$ is then a good quantum number, accounting for the quantization of the levels with increasing energy. The main role of the perturbation in (8) is to shift the energy levels downwards (for $a_{1}>0$ ) by an amount which is proportional to $k_{z}^{2}$. This explains that, for a sufficiently large value of $a_{1}$, the levels start to accumulate in the low-energy regime, producing the picture shown in Fig. 15(b).

\section{CONCLUSION}

We have shown that the thin films of WSs have a regime where they develop an ordered sequence of Landau bands, with the holelike levels well separated from their electronlike counterparts. The parameters for which we find that regime in our model pertain, actually, to the experimentally relevant domain realized in materials like TaAs (or $\mathrm{Cd}_{3} \mathrm{As}_{2}$ and $\mathrm{Na}_{3} \mathrm{Bi}$ in the case of the Dirac semimetals). Deviations of the parameters from that regime may, however, compromise the appearance of that ordered sequence, leading to multiple crossings between electron- and holelike bands and therefore preventing the observation of conductance quantization from the edge states.

The Landau levels of the WS are made of states which have, in general, 3D support, with a significant probability density across the bulk of the thin films. This means that the usual arguments implying the topological protection of the 2D quantum Hall effect cannot be applied in the present context. We stress, however, that the flatness of the Landau levels may still guarantee the quantization of the Hall conductance, provided that the states contributing to the current are localized at the edges of the sample. In the setup in Fig. 1, the total current $I_{y}$ across the whole transverse section of the bar can be obtained from the expression in Eq. (5). The derivative $\partial \varepsilon / \partial k_{y}$ can be taken as zero except at the dispersive branches of the Landau bands. When the electron- and holelike bands are separated as in the sequence in Fig. 4(b), the integral in (5) is nothing but the difference between the respective chemical potentials $\varepsilon_{+}, \varepsilon_{-}$at the two opposite edges of the bar times the number $n$ of dispersive bands picked up at the boundaries of the integration. We have therefore that $I_{y}=n(e / h)\left(\varepsilon_{+}-\varepsilon_{-}\right)$, leading to the usual quantization rule of the Hall conductance $G=n\left(e^{2} / h\right)$.

We conclude that the thin films of WSs may provide suitable setups to observe the quantum Hall effect at strong transverse magnetic field. In this picture, the quantization properties rest on the fact that the electronic transport takes place at the edges of the thin film. Our analysis may be useful as a guide for a proper measurement of the edge currents, which may have quite different profiles at the lateral boundaries depending on the orientation of the line connecting the Weyl nodes with respect to the transverse magnetic field.

\section{ACKNOWLEDGMENTS}

We acknowledge financial support through Spanish Grants No. PGC2018-094180-B-I00 (MCIU/AEI/FEDER, EU), No. FIS2017-82260-P, and No. FIS2015-63770$\mathrm{P}$ (MINECO/FEDER, EU), CAM/FEDER Project No. S2018/TCS-4342 (QUITEMAD-CM), and CSIC Research Platform PTI-001.
[1] X. Wan, A. M. Turner, A. Vishwanath, and S. Y. Savrasov, Topological semimetal and Fermi-arc surface states in the electronic structure of pyrochlore iridates, Phys. Rev. B 83, 205101 (2011).

[2] A. A. Burkov and L. Balents, Weyl Semimetal in a Topological Insulator Multilayer, Phys. Rev. Lett. 107, 127205 (2011).

[3] Z. K. Liu, B. Zhou, Y. Zhang, Z. J. Wang, H. M. Weng, D. Prabhakaran, S.-K. Mo, Z. X. Shen, Z. Fang, X. Dai, Z. Hussain, and Y. L. Chen, Discovery of a three-dimensional topological Dirac semimetal, $\mathrm{Na}_{3} \mathrm{Bi}$, Science 343, 864 (2014).

[4] M. Neupane, S. Xu, R. Sankar, N. Alidoust, G. Bian, C. Liu, I. Belopolski, T.-R. Chang, H.-T. Jeng, H. Lin, A. Bansil, F. Chou, and M. Z. Hasan, Observation of a three-dimensional topological Dirac semimetal phase in high-mobility $\mathrm{Cd}_{3} \mathrm{As}_{2}$, Nat. Commun. 5, 3786 (2014).

[5] S. Borisenko, Q. Gibson, D. Evtushinsky, V. Zabolotnyy, B. Büchner, and R. J. Cava, Experimental Realization of a 
Three-Dimensional Dirac Semimetal, Phys. Rev. Lett. 113, 027603 (2014).

[6] S.-Y. Xu, I. Belopolski, N. Alidoust, M. Neupane, G. Bian, C. Zhang, R. Sankar, G. Chang, Z. Yuan, C.-C. Lee, S.-M. Huang, H. Zheng, J. Ma, D. S. Sanchez, B. Wang, A. Bansil, F. Chou, P. P. Shibayev, H. Lin, S. Jia, and M. Z. Hasan, Discovery of a Weyl fermion semimetal and topological Fermi arcs, Science 349, 613 (2015).

[7] A. A. Burkov, Topological semimetals, Nat. Mater. 15, 1145 (2016).

[8] C. Fang, H. Weng, X. Dai, and Z. Fang, Topological nodal line semimetals, Chin. Phys. B 25, 117106 (2016).

[9] K.-Y. Yang, Y.-M. Lu, and Y. Ran, Quantum Hall effects in a Weyl semimetal: Possible application in pyrochlore iridates, Phys. Rev. B 84, 075129 (2011).

[10] A. C. Potter, I. Kimchi, and A. Vishwanath, Quantum oscillations from surface Fermi arcs in Weyl and Dirac semimetals, Nat. Commun. 5, 5161 (2014).

[11] H.-Z. Lu, S.-B. Zhang, and S.-Q. Shen, High-field magnetoconductivity of topological semimetals with short-range potential, Phys. Rev. B 92, 045203 (2015).

[12] J. Cao, S. Liang, C. Zhang, Y. Liu, J. Huang, Z. Jin, Z.-G. Chen, Z. Wang, Q. Wang, J. Zhao, S. Li, X. Dai, J. Zou, Z. Xia, L. $\mathrm{Li}$, and F. Xiu, Landau level splitting in $\mathrm{Cd}_{3} \mathrm{As}_{2}$ under high magnetic fields, Nat. Commun. 6, 7779 (2015).

[13] C. M. Wang, H.-P. Sun, H.-Z. Lu, and X. C. Xie, 3D Quantum Hall Effect of Fermi Arcs in Topological Semimetals, Phys. Rev. Lett. 119, 136806 (2017).

[14] The Fermi surface and quantum oscillations in thin films of Weyl semimetals with in-plane magnetic field were also studied by D. Bulmash and X.-L. Qi, Quantum oscillations in Weyl and Dirac semimetal ultrathin films, Phys. Rev. B 93, 081103(R) (2016).

[15] R. A. Molina and J. González, Surface and 3D Quantum Hall Effects from Engineering of Exceptional Points in Nodal-Line Semimetals, Phys. Rev. Lett. 120, 146601 (2018).

[16] C.-L. Zhang, S.-Y. Xu, C. M. Wang, Z. Lin, Z. Z. Du, C. Guo, C.-C. Lee, H. Lu, Y. Feng, S.-M. Huang, G. Chang, C.-H. Hsu, H. Liu, H. Lin, L. Li, C. Zhang, J. Zhang, X.-C. Xie, T. Neupert, M. Z. Hasan, H.-Z. Lu, J. Wang, and S. Jia, Magnetic-tunnelinginduced Weyl node annihilation in TaP, Nat. Phys. 13, 979 (2017).

[17] B. J. Ramshaw, K. A. Modic, A. Shekhter, Y. Zhang, E.-A. Kim, P. J. W. Moll, M. D. Bachmann, M. K. Chan, J. B. Betts, F.
Balakirev, A. Migliori, N. J. Ghimire, E. D. Bauer, F. Ronning, and R. D. McDonald, Quantum limit transport and destruction of the Weyl nodes in TaAs, Nat. Commun. 9, 2217 (2018).

[18] A theoretical analysis of this phenomenon based on ab initio calculations was also reported in P. Kim, J. H. Ryoo, and C.-H. Park, Breakdown of the Chiral Anomaly in Weyl Semimetals in a Strong Magnetic Field, Phys. Rev. Lett. 119, 266401 (2017).

[19] C. Zhang, Y. Zhang, X. Yuan, S. Lu, J. Zhang, A. Narayan, Y. Liu, H. Zhang, Z. Ni, R. Liu, E. S. Choi, A. Suslov, S. Sanvito, L. Pi, H.-Z. Lu, A. C. Potter, and F. Xiu, Quantum Hall effect based on Weyl orbits in $\mathrm{Cd}_{3} \mathrm{As}_{2}$, Nature (London) 565, 331 (2019).

[20] C. Fang, Y. Chen, H.-Y. Kee, and L. Fu, Topological nodal line semimetals with and without spin-orbital coupling, Phys. Rev. B 92, 081201(R) (2015).

[21] H. Weng, C. Fang, Z. Fang, B. A. Bernevig, and X. Dai, Weyl Semimetal Phase in Noncentrosymmetric TransitionMetal Monophosphides, Phys. Rev. X 5, 011029 (2015).

[22] Y. Kim, B. J. Wieder, C. L. Kane, and A. M. Rappe, Dirac Line Nodes in Inversion-Symmetric Crystals, Phys. Rev. Lett. 115, 036806 (2015)

[23] R. Yu, H. Weng, Z. Fang, X. Dai, and X. Hu, Topological NodeLine Semimetal and Dirac Semimetal State in Antiperovskite $\mathrm{Cu}_{3}$ PdN, Phys. Rev. Lett. 115, 036807 (2015).

[24] We recall that the Fermi arcs of Weyl semimetals can also be understood as the zeroth Landau level of an axial pseudomagnetic field confined to the surface, as discussed by A. G. Grushin, J. W. F. Venderbos, A. Vishwanath, and R. Ilan, Inhomogeneous Weyl and Dirac Semimetals: Transport in Axial Magnetic Fields and Fermi Arc Surface States from Pseudo-Landau Levels, Phys. Rev. X 6, 041046 (2016).

[25] Fermi-arc-mediated transport in semimetal nanowires was also recently considered by V. Kaladzhyan and J. H. Bardarson, Quantized Fermi arc mediated transport in Weyl semimetal nanowires, Phys. Rev. B 100, 085424 (2019).

[26] H.-P. Sun and H.-Z. Lu, Quantum transport in topological semimetals under magnetic fields (II), Front. Phys. 14, 33405 (2019).

[27] This may significantly prevent the scattering of surface states into the bulk, studied originally by E. V. Gorbar, V. A. Miransky, I. A. Shovkovy, and P. O. Sukhachov, Origin of dissipative Fermi arc transport in Weyl semimetals, Phys. Rev. B 93, 235127 (2016). 